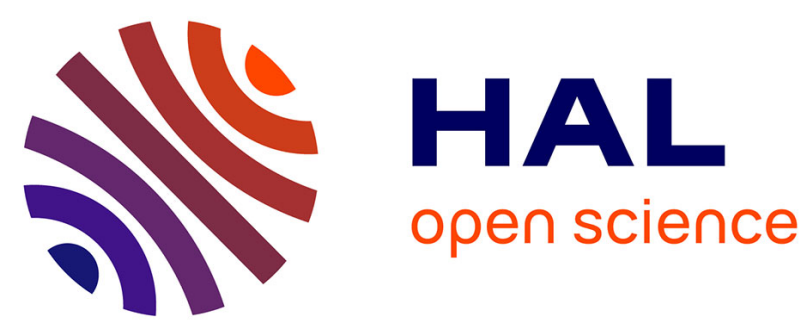

\title{
Bis-3,5-Diamino-1,2,4-Triazolyl-1,2,4,5-Tetrazine: From Insensitive High Energy Density Materials to Small Molecule Organic Semiconductors
}

Artem Babaryk, Mohamed Haouas, Olena Khaynakova, Erik Elkaïm, Patricia Horcajada

\section{To cite this version:}

Artem Babaryk, Mohamed Haouas, Olena Khaynakova, Erik Elkaïm, Patricia Horcajada. Bis3,5-Diamino-1,2,4-Triazolyl-1,2,4,5-Tetrazine: From Insensitive High Energy Density Materials to Small Molecule Organic Semiconductors. Crystal Growth \& Design, 2020, 20 (10), pp.6510-6518. 10.1021/acs.cgd.0c00698 . hal-03000889

\section{HAL Id: hal-03000889 https://hal.science/hal-03000889}

Submitted on 12 Nov 2020

HAL is a multi-disciplinary open access archive for the deposit and dissemination of scientific research documents, whether they are published or not. The documents may come from teaching and research institutions in France or abroad, or from public or private research centers.
L'archive ouverte pluridisciplinaire HAL, est destinée au dépôt et à la diffusion de documents scientifiques de niveau recherche, publiés ou non, émanant des établissements d'enseignement et de recherche français ou étrangers, des laboratoires publics ou privés. 


\section{Bis-3,5-Diamino-1,2,4-Triazolyl-1,2,4,5-Tetrazine: From Insensitive High Energy Density Materials to}

\section{Small Molecule Organic Semiconductors}

Artem A. Babaryk', Mohamed Haouas ${ }^{2}$,Olena A. Khaynakova ${ }^{3}$ Erik Elkäim ${ }^{4}$, Patricia Horcajada

'Advanced Porous Materials Unit (APMU), IMDEA Energy, Avda. Ramón de la Sagra 3, E28935 Móstoles, Madrid, Spain

${ }^{2}$ Institut Lavoisier de Versailles, UVSQ, University of Paris-Saclay, 45 Avenue des Etats-Unis, 78035, Versailles Cedex, France

32Departamento de Química Física y Analítica, Universidad de Oviedo, Julián Clavería 8, Oviedo 33006, Spain

${ }^{4}$ CRISTAL beamline. Synchrotron SOLEIL, L'Orme des Merisiers, Saint-Aubin, BP 48, 91192 Gif-sur-Yvette Cedex, France

High Energy Density Material, Small Molecule Organic Semiconductor, Crystal Structure, Powder X-ray Diffraction, Solid State Nuclear Magnetic Resonance

ABSTRACT 
Besides the well-known pyrotechnic applications of insensitive high energy compounds such as $s$-tetrazine, they are promising materials for relevant applications (e.g. electrochemical energy storage, photocatalysis). Bis-3,5-diamino-1,2,4-triazolyl-1,2,4,5-tetrazine (BDTT) is a nitrogenrich conjugated heterocyclic compound $(\mathrm{N} \sim 71 \mathrm{wt} \%)$, inert to ignition and mechanical stimuli and thermally stable up to $370{ }^{\circ} \mathrm{C}$. The solvent-free crystalline structure of this high energy density compound (named IEF-12 for IMDEA Energy framework) is reported here for the first time using the high resolution powder X-ray diffraction (PXRD) and ${ }^{\prime} \mathrm{H},{ }^{\mathrm{i}} \mathrm{C}$, and ${ }^{\mathrm{i}} \mathrm{N}$ solid state nuclear magnetic resonance (NMR) spectroscopy. We demonstrate that IEF-12 has features of small molecule organic semiconductor, namely, lamellar $\pi$-stacked crystal packing in solid state,

low energy optical bandgap $=2.27 \mathrm{eV}$ and relatively high value of dielectric constant $\varepsilon_{\mathrm{r}}=$ 4.86. Overall, we exemplified on EIF-12 that insensitive high energy density materials (HEDM) may open an avenue for non-classically structured organic semiconductors, paving the way for optoelectronic and photovoltaic applications.

\section{INTRODUCTION}

Electron deficiency of 1,2,4,5-tetrazine or, more commonly, $s$-tetrazine results in unusual chemical and physical properties of generic compounds. In particular, 3,6-substituted $s$-tetrazines with a high-nitrogen content have attracted significant attention from researchers, due to their potential use as high energy density materials (HEDM) in civil and military pyrotechnic domains (e.g. automotive airbags, fireplaces, safety matches, oxygen candles, fireworks, igniters, ammunitions, gas-pressure blasting in mining, quarrying, and demolition). This circumstance somewhat dwarfs other uses of $s$-tetrazine molecular materials for different industrially and societally relevant application domains. For instance, $s$-tetrazines can reversibly be reduced to stable anion-radicals, making them promising materials for electrochemical energy storage. ${ }^{2}$ From the other hand, they exhibit a well-defined $\pi-\pi^{*}$ transition located at the UV region, which 
energy depends on the nature of 3,6-substituents. Coupling of both properties is successfully utilized for the fabrication of electroactive polymeric materials ${ }^{4}$ and applied for photocatalysis of organic reactions..$^{5.6}$

Despite their inherent instable character, the conjugation of azole rings with the tetrazine core at para-position leads to remarkably high stable derivatives. For instance, Chavez \& Hiskey reported the synthesis and propellant properties of the bis(tetrazolylaminotetrazine), with a low impact sensitivity, non-explosive, non-pyrotechnic and nonflammable character. Kläpotke et al. . $^{\text {. }}$ explored the nucleophilic substitution in s-tetrazine using high electrophilic properties of 3,6dichloro-1,2,4,5-tetrazine to obtain a series of five new HEDMs. Amongst them, bis-3,5diamino-1,2,4-triazolyl-1,2,4,5-tetrazine (BDTT) was distinguished by its outstanding thermal stability $\left(>370{ }^{\circ} \mathrm{C}\right)$. Further, BDTT has one order of magnitude greater heat of formation than the well-known space rocket propellant hexanitrostilbene, owing to the presence of highly endothermic $\mathrm{N}-\mathrm{N}$ and $\mathrm{C}-\mathrm{N}$ bonds, as it was assumed. However, it demonstrates 8-fold less sensitivity towards impact stimulus, probably due to an absence of any exothermic bonds as well.

From 80 s, undoped organic semiconductors have been attracting the researchers' interest due to their application in photovoltaic cells, incorporating an organic hetero-junction of $p$ - and $n$ conducting materials, organic light-emitting devices (OLEDs) or logic circuits with organic field-effect transistors (OFETs) $n$-conducting materials. It has been shown that small molecules with acceptor-donor-acceptor structure, containing electron-accepting tetrazine moiety and electron-donating oligothiophenes, could be used as components of organic photovoltaic solar cells with efficacies as high as $3.24 \%$ at $J_{s c}$ of $7.87 \mathrm{mAcm}^{2}{ }^{\circ} n$-Semiconducting halophenyltriazines were computationally and experimentally studied, yielding to relatively moderate charge mobilities $\left(0.006-0.026 \mathrm{~cm}^{2} \mathrm{~V}^{-1} \mathrm{~s}^{-1}\right)$, that could be improved by switching to bis(aryleneethynylene)-s-tetrazines (up to $\left.0.3-1.3 \mathrm{~cm}^{2} \mathrm{~V}^{-1} \mathrm{~s}^{-1}\right) .{ }^{1 "}$ Note here that the latter values are very promising on light organic photovoltaics.

BDTT crystal structure (named IEF-12, IEF for IMDEA Energy Framework) was obtained as a bulk dark-red precipitate, exhibiting optical absorption in the range of 1.5-2.5 eV. These values 
are typical for $\pi-\pi^{*}$ transitions in organic semiconducting materials (OSM) like, for instance in pentacene, a classical long-standing and well-studied representative OSM. Considering the fact that van der Waals forces have major importance for molecular packing in crystalline organic solids, ${ }^{12}$ the delocalization of electronic wavefunctions over adjacent molecules has important direct implications on charge carrier transport. Precisely, the transfer integral value is responsible for degenerating of highest occupied molecular orbital (HOMO) and the lowest unoccupied molecular orbital (LUMO) into zones, similarly to conventional inorganic semiconductors. When a charge carrier passes through a molecule, it losses some portion of energy (reorganization energy) which, in due course, depends on the conjugation length, degree, and packing of the organic molecules in the crystal. The very low solubility of EIF-12 suggests the presence of strong intermolecular interactions, contributing to the general cohesion of the crystal and, hence, knowledge of the spatial organization is a precondition for the rational selection of OSMs for optoelectronic applications. The typical fashions of $\pi$-bonded systems arrangement are schematically given in Figure 1. It is believed that the most efficient arrangement, in terms of the higher charge transfer integral and the lesser reorganization energy, is the gliding $\pi$-stacked lamellar motif (Fig. 1d).

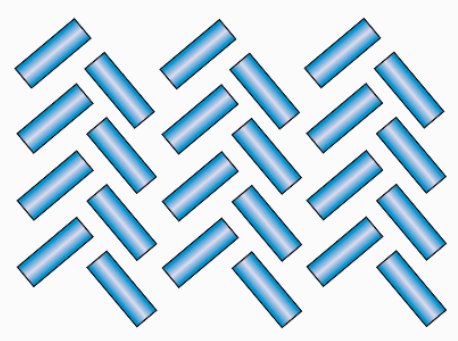

a

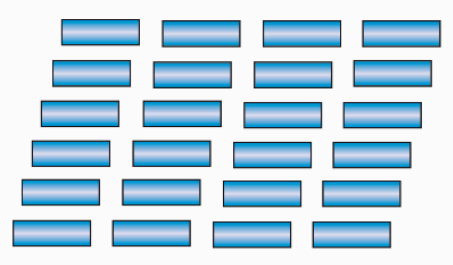

C

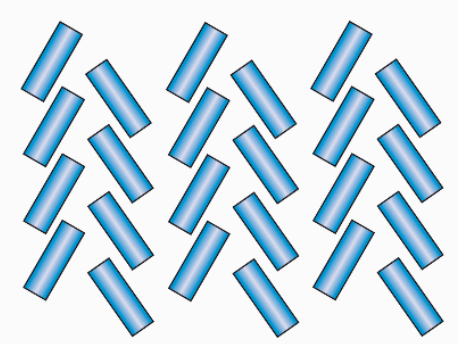

b

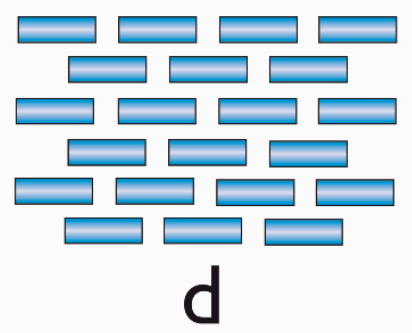


Figure 1. Usual schematic crystal packing types of OSMs: (a) herringbone packing (face-toedge) without $\pi-\pi$ overlap (face-to-face) between adjacent molecules; (b) herringbone packing with $\pi-\pi$ overlap between adjacent molecules; (c) lamellar motif, parallel $\pi$-stacking; (d) lamellar motif with gliding $\pi$-stacking.

In this paper, the synthesis of IEF-12 structure was successfully replicated to elucidate the spatial organization and bulk electronic properties, proving to be interesting candidates not only for traditional HEDM application fields (pyrotechnics), but also for emerging OSMs (e.g. OLEDs, OFETs, solar cells). We report the accurate $a b$ initio determination of the BDTT crystal structure (IEF-12) by high resolution powder $\mathrm{X}$ ray diffraction (PXRD) and ${ }^{\mathrm{H}} \mathrm{H},{ }^{\mathrm{I}} \mathrm{C}$, and ${ }^{\mathrm{s}} \mathrm{N}$ solid state nuclear magnetic resonance (NMR). IEF-12 structure is intrinsically lamellar due to the net of strongly $\mathrm{H}$-bonded molecules, facilitating additional $\pi$-bonding between lateral donor amino groups of tetrazolyl substituent and tetrazine core acceptor. This is the first structural determination of $s$-tetrazine based HEMD, free of crystallization solvent. We put into agreement the optical bandgap measured by UV-vis spectroscopy with electronic band structure calculations. In addition, the static dielectric constant of IEF-12 was determined by the density functional perturbation theory calculations calibrated on the Fourier transformed infrared (FTIR) and Raman spectroscopies. Further, we rationalize here the low solubility of the compound from the post-refinement crystal structure analysis and establish a tentative charge transfer pathway from the interplay of solid and solute state (wherever it is possible) using a combination of UVvis and $\mathrm{H}$ NMR spectroscopies in solution and time-dependent density functional theory (TDDFT) molecular simulations.

\section{MATERIALS AND METHODS}

Synthesis. IEF-12 was synthesized using a slightly modified protocol previously reported by Kläpotke et al. ${ }^{\circledR}$ Briefly, 3,5-diamino-1,2,4-triazole (DATR; $1 \mathrm{~g}, 10.08 \mathrm{mmol}$ ) was dissolved in 25 $\mathrm{mL}$ of $N, N^{\prime}$-dimethylformamide (DMF) at a room temperature (RT) using extensive alternate 
sonication and stirring until bulky crystals of DATR completely disappeared, yielding paleyellow solution. Then, a solution of dichlorotetrazine $(0.377 \mathrm{~g}, 2.50 \mathrm{mmol})$ in $5 \mathrm{~mL}$ of acetonitrile was added dropwisely for $0.5 \mathrm{~h}$. The product started to precipitate immediately. The suspension was stirred for $2 \mathrm{~h}$ at RT and then, refluxed at $120^{\circ} \mathrm{C}$ overnight. After filtration, the product was successively washed with large amounts of DMF (about $200 \mathrm{~mL}$, until the washing residues turned completely colorless), $150 \mathrm{~mL}$ of distilled water and $150 \mathrm{~mL}$ of acetonitrile to obtain $0.9 \mathrm{~g}(5.07 \mathrm{mmol}$, yield $\sim 86 \mathrm{wt} \%)$ of IEF-12, a deep-red fine powder as it was confirmed by scanning electron microscopy (SEM; see Figure S1 in the Supporting Information-SI). Final product was dried at open air and then, dried at $60^{\circ} \mathrm{C}$ in an air-ventilated oven.

Powder X-ray diffraction (PXRD). About $50 \mathrm{mg}$ of IEF-12 were loaded in a $1.0 \times 20 \mathrm{~mm}$ borosilicate glass capillary (Hilgenberg $\mathrm{GmbH}$ ). The data were collected in the parallel beam geometry using a Bruker D8 Advance system equipped with $\mathrm{Cu}$-anode tube, Göbbel mirror, 0.2 mm collimating slit and LynxEye PSD detector, at ambient conditions. The variable counting time strategy ${ }^{13}$ was chosen to improve the quality of the experimental data for the structure determination process ${ }^{14}$ and statistically meaningful dataset of $\sin \Theta \cdot \lambda^{-1}=0.77 \AA^{-1}$ resolution was collected.

The high-resolution synchrotron data were collected at the CRISTAL beamline (SOLEIL, Saint-Aubin, France) with Si(111)-monochromated beam and the wavelength of $\lambda=0.7289 \AA$. The capillary specimen was mounted on a 2-circle diffractometer and linear PSD Mythen (PSI, Switzerland) detector at RT.

UV-vis Spectroscopy. UV-vis data were collected using a Perkin-Elmer spectrometer equipped with a tungsten-krypton light source. The spectra of dimethylsulfoxide (DMSO) solutions $\left(1 \times 10^{\urcorner} \mathrm{M}\right)$ were recorded at a RT (10 $\mathrm{mm}$ path length). Solid state measurements were performed relatively to $\mathrm{BaSO}_{4}$ using a Praying Mantis attachment with an integrating sphere to measure diffuse reflectance. The measurements on the powder sample consisted of a small amount of powder pressed into a micro sample holder, which was exposed to light of 250-800 nm wavelengths segment. The Kubelka-Munk function $F(R)$ was calculated from the diffuse 
reflectance measurements $R$, using the equation $F(R)=(1-R)^{2}(2 R)^{-1}$. Tauc plots were then plotted using $(h v F(R))^{n}=f(h v)$ where $n=1 / 2$ and 2 for direct and indirect transitions, respectively.

Solid State Nuclear Magnetic Resonance. Solid-state NMR spectra were recorded on Bruker Avance II spectrometer equipped with a $11.7 \mathrm{~T}$ wide-bore superconducting magnet. Samples were packed into $2.5 \mathrm{~mm}$ zirconia rotors and rotated at the magic angle spinning (MAS) at 30 $\mathrm{kHz}$ for ${ }^{\mathrm{H}} \mathrm{H}$ or at $10 \mathrm{KHz}$ for ${ }^{\mathrm{i}} \mathrm{C}$ and ${ }^{15} \mathrm{~N}$. ${ }^{\mathrm{H}} \mathrm{NMR}$ spectra were recorded using one-pulse sequence with signal averaging for 8 transients with a repeat interval of $55 \mathrm{~s}$. ${ }^{18} \mathrm{C}$ and ${ }^{15} \mathrm{~N}$ NMR spectra were recorded using cross-polarization $(\mathrm{CP})$ from $\mathrm{H}$ with a spin lock pulse (ramped for $\mathrm{H}$ ) of $10 \mathrm{~ms}$. SPINAL-64 decoupling of ${ }^{\mathrm{H}}\left(\boldsymbol{v}_{1}=50 \mathrm{kHz}\right)$ was applied during acquisition. Signal averaging was carried out for 1024 transients for ${ }^{13} \mathrm{C}$ and 8704 transients for ${ }^{15} \mathrm{~N}$ with a repeat interval of $7 \mathrm{~s}$. Chemical shifts are reported relative to tetramethylsilane (TMS; $\mathrm{H}$ and $\left.{ }^{13} \mathrm{C}\right)$ and nitromethane $\left.\mathrm{CH}_{3} \mathrm{NO}_{2} ;{ }^{15} \mathrm{~N}\right)$, using secondary solid references of adamantane $\left(\mathrm{CH}_{2}=1.7 \mathrm{ppm}\right.$, and $\mathrm{CH}_{2}=38.5$ ppm) and histidine $\left(\mathrm{N}_{2}=-212.0 \mathrm{ppm}\right)$.

Vibrational Spectroscopy. Attenuated total reflection Fourier transform infrared (ATR-FTIR) spectrum was recorded using a Nicolet 6700 instrument. Three consequent scans were collected with 128 accumulations per scan in a range of $500-4000 \mathrm{~cm}^{-1}$ and averaged to minimize experimental signal errors.

Raman spectra were recorded with a JASCO NRS-5100 confocal Raman microscope using glass plate. A Nd:YAG laser excitation was operated at $0.2 \mathrm{~mW}$ power and Stokes Raman scattering was monitored with a Peltier cooled CCD detector in the range of 50-3650 $\mathrm{cm}^{-1}$.

\section{Computational details.}

Gas Phase Calculations. Unconstrained structures of geometric isomers were optimized using density functional theory (DFT) method B3LYP hybrid functionalis together with 6-311++G(d,p) Pople basis set. ${ }^{16}$ Frequency calculations were performed following geometry optimizations to ensure minimum-energy structures. Orbital and spectral predictions included the solute molecule density model (SMD) for dimethylsulfoxide (DMSO) solvation $(\varepsilon=47.2)$. All the excitation energies were converged at the cc-pVQZ basis set. ${ }^{17} \mathrm{H},{ }^{13} \mathrm{C}$, and ${ }^{15} \mathrm{~N}$ NMR shifts were calculated at 
def2-TZVPP basis set. ${ }^{18}$ All the calculations were carried out using the Orca 4.0 program. ${ }^{19}$ The Avogadro program was used to edit xyz-files.20

Periodic Boundary Phase Calculations. Periodic boundary density functional theory (PBDFT) calculations were carried out using the plane-wave pseudopotential method, as implemented in the CASTEP code. ${ }^{21}$ Initial coordinates of atoms in the unit cell were taken from PXRD refined crystal structure and used for further optimizations. Generalized gradient approximation of DFT in Perdew-Becke-Ernzerhof parametrization ${ }^{22}$ was applied to account for electron exchange and correlational. Van der Waals interactions were accounted with aid of Tkatchenko-Scheffler method. ${ }^{23}$ The plane-wave cutoff energy was defined as $1200 \mathrm{eV}$ upon tests of convergence of self-consistent field energy calculations using on-the-fly generated normconserving pseudopotentials. ${ }^{2425}$ Brillouin zone sampling of electronic states was performed on $6 \times 4 \times 4$ Monkhorst-Pack grid. The structure at equilibrium, an essential prerequisite for lattice dynamics calculations, was established by Broyden-Fletcher-Goldfarb-Shanno algorithm geometry optimization, after which the residual forces were converged to $<10^{-2} \mathrm{eV} \AA^{-1}$. Phonon frequencies were obtained by diagonalization of dynamical matrices computed using density functional perturbation theory (DFPT). ${ }^{20.27}$ The determination of solid-state NMR parameters was performed with aid of the GIPAW method ${ }^{28}$ The same as given for above control parameters were used for computations of absolute chemical shielding. The predicted chemical shifts were calculated using the relationship $\delta_{\mathrm{sis}}=\sigma_{\mathrm{ref}}-\sigma_{\mathrm{sos}}$ where $\sigma_{\mathrm{ref}}$ corresponds to magnetic shielding for the reference compound and $\sigma_{\mathrm{is}}=\left(\sigma_{11}+\sigma_{22}+\sigma_{33}\right) / 3$. The magnetic shielding for ${ }^{1} \mathrm{H},{ }^{13} \mathrm{C}$, and ${ }^{15} \mathrm{~N}$ signal reference compounds were set at 30.97, 171 (TMS) and -154.3 ppm $\left(\mathrm{CH}_{3} \mathrm{NO}_{2}\right)_{.^{29}}$

\section{RESULTS AND DISCUSSION}

X-ray Structure Analysis. Remarkably, to the best of our knowledge, the IEF-12 crystal structure is the first example of a s-tetrazine based polynitrogen HEDM determined in its pristine state. Other derivatives were isolated solely at the solvated state, typically with the inclusion of 
DMSO molecules..$^{30.32}$ The presently reported material does not exhibit $(\mathrm{S}=\mathrm{O})$ vibrational modes in an agreement with referenced infrared (IR) observations of Kläpotke et al., characteristic of DMSO, ruling out the solvated crystal hypothesis (as we will show further). From basic considerations, BDTT molecule could appear as one of two possible geometrical isomers, in trans or cis configuration (Figure 2 and Table S1-S2). To account for both alternative structures, we constructed initial molecular models and optimized their geometries in vacuo.

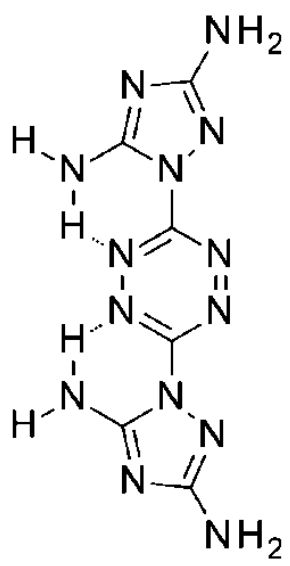

cis-isomer<smiles></smiles>

trans-isomer

Figure 2. Probable (left) cis- and (right) trans-isomers of BDTT.

PXRD pattern of IEF-12 was successfully indexed using singular value decomposition algorithm in anorthic cell setting $\left(\mathrm{SVD} \mathrm{M}_{23}=21\right),{ }^{33}$ with the final unit cell parameters after converged Pawley pattern decomposition $\left(\mathrm{R}_{\mathrm{vp}}=3.5, \mathrm{GoF}=2.1\right): a=5.2707(5) \AA, b=6.8529(7)$ $\AA, c=8.0771(7) \AA, \alpha=97.050(6)^{\circ}, \beta=104.964(7)^{\circ}, \gamma=100.692(5)^{\circ}, V=272.44(5) \AA^{3}$. For the given Laue symmetry class, the highest possible set of symmetries in anorthic class is for $P-1$ space group. Implying the presence of an inversion center in both geometries, a reasonable choice of the space group is $P-1$, albeit it is difficult to select properly from $P 1$ or $P-1$ basing solely on powder diffraction data. From the ${ }^{13} \mathrm{C}$ and ${ }^{15} \mathrm{~N}$ solid state NMR experiments (Figure 4; see below), two independent signals of carbon and seven signals of nitrogen are detected, which indicates that half of the complete molecule resides in the asymmetric unit cell $\left(Z^{\prime}=0.5\right.$; Scheme $\mathrm{S} 1)$. The structure solution was performed using direct space methods.$^{34}$ Only $(\mathrm{N} 1-\mathrm{C} 1-\mathrm{N} 3-\mathrm{C} 2)$ or 
(N1-C1-N3-N5) torsion angles (see Figure 4d or Scheme 1 for the numeration), corresponding to conjugated tetrazine core and triazole cycles rotation across $\mathrm{C}-\mathrm{C}$ axis, were used as internal molecular degrees of freedom (translations and rotations of a rigid body taken as an entire during simulated annealing cycles). The best solution was obtained at $w R=10.5 \%$, based on the Rietveld's definition for the cost functions of solution quality. It is worth to note that employing both initial geometries led to a unique solution, in favor of the trans-isomer. Initial crystal atomic coordinates were refined using Rietveld method $\left(R_{\mathrm{wp}}=4.12 \%, R_{\mathrm{p}}=3.19 \%, \mathrm{GoF}=4.03\right.$, see SI, Figure S2 and Table S3 for further details).

Principally, molecular conformation of BDTT ought to adopt planar configuration due to the conjugation of electron donating properties of guanazole cycle ${ }^{35}$ with electron-deficient $\pi$-system of tetrazine. Expectedly, the final interplanar angle value between the tetrazine and triazole ring is equal to $4.6(9)^{\circ}$. The donor-acceptor-donor (D-A-D) type of molecule strongly affects the intra- and inter-molecular hydrogen bond system. The hydrogen bond net can be efficiently rationalized using a graph set description (SI). The closer amino group in third position of triazole ring forms two symmetric $\mathrm{S}(6)$ rings (here and after, we will use notations as given in ref. 36 of intramolecular $\mathrm{H}$-bonds with participation of $\mathrm{N}$-acceptor atoms of tetrazine core; see detailed description in SI).

It is known that a crystal packing of a $\pi$-conjugated system defines the performance of organic semiconductors, affecting the transfer integral and reorganization energy of molecules. In the present case, the dense formed hydrogen bond net favors desirable lamellar motif of packed molecules: i.e., free hydrogen atom of the same terminal amino-group is engaged into $\mathrm{R}_{2}^{2}(8)$ cyclic H-bonding with a molecule of the next layer (Figure 3a). Essentially, the same type of cycle occurred with participation of the amino-group at $\mathrm{C} 2$ of triazolyl ring through the in-plane head-to-tail arrangement, as can be seen from Figure 3b. The particular D-A-D molecular organization has an impact on crystal structuring enabling non-covalent interactions of electron enriched terminal amino-groups as $\sigma$-donors with electron poor tetrazine $\pi$-acceptor rings at a distance of $2.944 \AA$. 


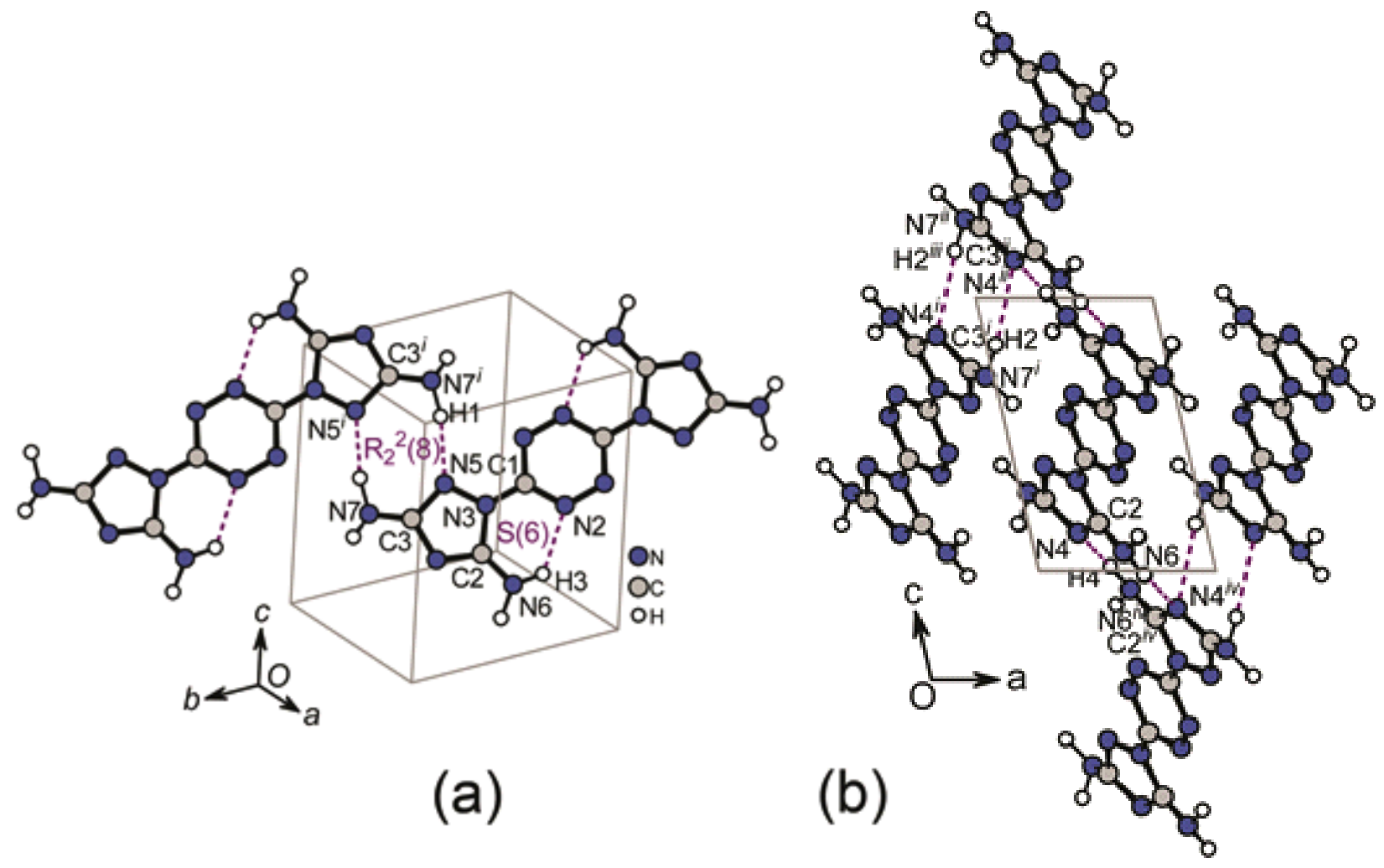

Figure 3. View of the (a) lateral head-to-tail H-bonded ensembles, and (b) vertical inter-sheet interaction of BDTT molecules. Symmetry codes: $(i)-x, 1-y, 1-z ;(i i): x, y, 1+z ;(i i i):-x, 1$ $-\mathrm{y}, 2-\mathrm{z} ;(i v): 1-\mathrm{x}, 1-\mathrm{y},-\mathrm{z}$.

A 2D $\pi$-stacked lamellar motif of the crystal building oughts to be the most efficient for the charge transport, ${ }^{12}$ and hence, the IEF-12 crystal structure is a good candidate for further charge transport studies. Following this, herewith we focus our attention on the strongest interacting parts of the crystalline lattice, which could be representative molecular aggregates for charge transfer.

The empirical "UNI" pairwise interatomic potential parameters ${ }^{37}$ allow to identify the strongest interactions between molecular aggregates in the crystal for $\mathrm{C}, \mathrm{H}, \mathrm{N}, \mathrm{O}$ species by quantifying the inter-molecular potentials in a crystal. Two molecules ensemble supported with nitrogen- $\pi$ contacts are stacked along the $b$-axis (Figure S3) at a distance of $6.79 \AA$ with the packing energy (PE) of $59.95 \mathrm{~kJ} \mathrm{~mol}^{-1}$, while corresponding value for in-plain H-bonded aggregate is only 47.34 $\mathrm{kJ} \mathrm{mol}^{-1}$. Therefore, interaction strength at distances equal to separations between tetrazine core 
centroids strongly depends on the direction of intermolecular interactions. Finally, PE per unit formula (calculated from a shell of 200 molecules) yields to $233.7 \mathrm{~kJ} \mathrm{~mol}^{-1}$, indicating a very strong cohesion of crystallized molecules. The packing energy often tightly correlates with $\Delta H_{\text {sub }}$ and thus, with $\varrho_{\text {×xp }}=1.75 \mathrm{~g} \mathrm{~cm}^{3}$ and low solubility $\left(10^{-5} \mathrm{M}\right.$ in DMSO at RT). Thus, one can suggest here a high-vacuum route for the shaping of this material as thin films (vs. wet deposition methods), as commonly used for insoluble conjugated organic semiconductors. ${ }^{38}$

Solid state NMR. To validate the structural model obtained from the XRD study, solid-state NMR spectroscopy was employed. Figure 4 presents the obtained ${ }^{1} \mathrm{H},{ }^{1} \mathrm{C}$ and ${ }^{15} \mathrm{~N}$ NMR spectra. Assignment of all signals is based on comparison with DFT calculations, which are summarized in Table 1.

I have a problem with the Table 1 content. Please revise: i) "calculated symmetric components of cs" where are they, $\delta 11, \delta 22, \delta 33$ or $\sigma 11, \sigma 22, \sigma 33$ ??? ii) efg tensors, where are they? Instead I see quadrupolar coupling, no sense !!!! Do you have Vxx, Vyy, Vzz ? In my opinion, put in Table 1: oiso (ppm), $\sigma 11$ (ppm), $\sigma 22$ (ppm), $\sigma 33(\mathrm{ppm}), \eta(\sigma 11-\sigma 22 / \sigma 33), \delta$ calc (ppm),

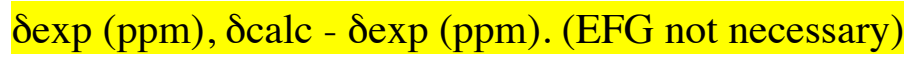

Table 1. Calculated symmetric components of chemical shielding, electric field gradient tensors, and chemical shifts, compared to experimental values from ${ }^{1} \mathrm{H},{ }^{\mathrm{I}} \mathrm{C}$, and ${ }^{\mathrm{s}} \mathrm{N}$ MAS data.

\begin{tabular}{ccccccccc}
\hline Atom & $\boldsymbol{\sigma}_{\text {iso }}(\mathbf{p p m})$ & $\boldsymbol{\sigma}_{\text {amis }}(\mathbf{p p m})$ & $\kappa$ & $\mathbf{C}_{\ell}(\mathbf{M H z})$ & $\eta$ & $\begin{array}{c}\boldsymbol{\delta}_{\text {alk }} \\
(\mathbf{p p m})\end{array}$ & $\begin{array}{c}\boldsymbol{\delta}_{\text {cep }} \\
(\mathbf{p p m})\end{array}$ & $\begin{array}{c}\boldsymbol{\delta}_{\text {calk }} \boldsymbol{\delta}_{\text {avp }} \\
(\mathbf{p p m})\end{array}$ \\
\hline $\mathbf{H 1}$ & 22.63 & 17.97 & 0.29 & 0.197 & 0.17 & 8.34 & 7.1 & 1.2 \\
$\mathbf{H 2}$ & 26.80 & 12.73 & 0.23 & 0.243 & 0.15 & 4.17 & 3.8 & 0.4 \\
$\mathbf{H 3}$ & 23.71 & 13.33 & 0.50 & 0.222 & 0.15 & 7.26 & 7.1 & 0.2 \\
$\mathbf{H 4}$ & 21.66 & 18.02 & 0.37 & 0.188 & 0.20 & 9.31 & 9.8 & -0.5 \\
$\mathbf{C 1}$ & 11.79 & -165.74 & 0.68 & -1.197 & 0.94 & 159.21 & 157.5 & 1.7 \\
$\mathbf{C 2}$ & 9.05 & 108.28 & 0.65 & 1.836 & 0.48 & 161.95 & 157.5 & 4.4 \\
$\mathbf{C 3}$ & 16.77 & 98.07 & 0.89 & -2.032 & 0.47 & 154.23 & 163.3 & -9.1 \\
\hline
\end{tabular}




\begin{tabular}{lcccccccc}
\hline $\mathbf{N 1}$ & -137.08 & 536.70 & 0.79 & -5.180 & 0.06 & -17.22 & -32.1 & 14.9 \\
$\mathbf{N 2}$ & -138.51 & 536.45 & 0.86 & -5.181 & 0.02 & -15.79 & -27.2 & 11.4 \\
$\mathbf{N 3}$ & 42.27 & 65.92 & 0.48 & -4.186 & 0.29 & -196.57 & -210.4 & 13.8 \\
$\mathbf{N 4}$ & 33.36 & 183.32 & 0.62 & 2.627 & 0.90 & -187.66 & -193.1 & 5.4 \\
$\mathbf{N 5}$ & 14.07 & -175.04 & 0.86 & 5.332 & 0.12 & -168.37 & -168.0 & -0.4 \\
$\mathbf{N 6}$ & 149.52 & -86.80 & 0.27 & -3.350 & 0.56 & -303.82 & -314.6 & 10.8 \\
$\mathbf{N 7}$ & 167.83 & -74.97 & 0.25 & -3.840 & 0.41 & -322.13 & -331.9 & 9.8 \\
\hline
\end{tabular}

The H MAS NMR exhibits a composite spectrum with at least three overlapped peaks with apparent maxima at 3.8 and $7.1 \mathrm{ppm}$ and a shoulder at $c a .9 .8 \mathrm{ppm}$. The calculated isotropic shifts for "inactive" N-H bond is $4.2 \mathrm{ppm}$, falling into $\pm 0.5 \mathrm{ppm}$ accuracy of proton shift, determined with GIPAW. The signal from H-atoms takes part in the intramolecular hydrogen bonds, being observed at 7.3 and $8.3 \mathrm{ppm}$. However, that difference is probably experimentally smaller, resulting in their merging and leading to observed peak integral accounting for two protons. The hint on the shoulder at $9.8 \mathrm{ppm}$ should be associated with a poorly resolved low field peak of strong intermolecular $\mathrm{H}$-bonded protons having the shortest $\mathrm{H}-\mathrm{N}$ distances $(<2$ A). 

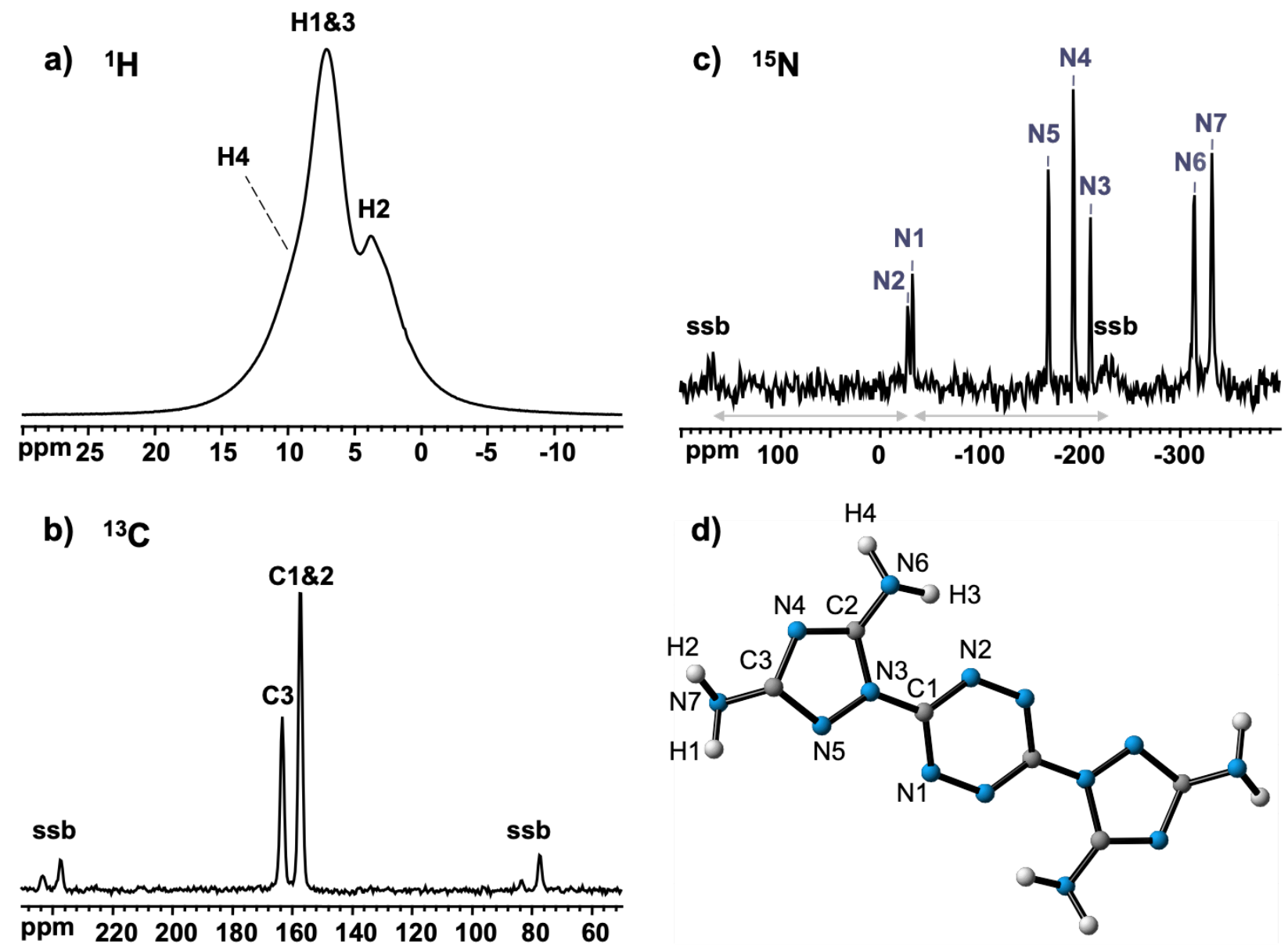

Figure 4. (a) 'H MAS, (b) ${ }^{18} \mathrm{C}\left\{{ }^{\prime} \mathrm{H}\right\}$ CPMAS, and (c) ${ }^{15} \mathrm{~N}\left\{{ }^{\prime} \mathrm{H}\right\}$ CPMAS spectra of IEF-12 solid. (d) Molecular structure of BDTT in the crystal packing, showing the inequivalent atom labels used for spectral assignment according to the DFT-GIPAW calculations. "ssb" denotes spinning side bands.

${ }^{13} \mathrm{C}$ and ${ }^{15} \mathrm{~N}$ solid state NMR signals bring chemically rich information about IEF-12 molecular packing. Consistent with $Z^{\prime}=0.5$, all seven expected signals of nitrogen are observed, while only two over the three expected signals of carbon are visible. The C3 carbon of triazole is the most shielded $\left(\sigma_{\mathrm{is}}=16.8 \mathrm{ppm}, \Delta \delta=3.9 \mathrm{ppm}\right.$, where $\left.\Delta \delta=\sigma_{33}-\sigma_{\mathrm{iso}}\right)$, while the signals of $\mathrm{C} 1$ and C2 differ slightly by less than $3 \mathrm{ppm}$ and are experimentally unresolved. The latter has even somewhat smaller magnetic shielding than the former that may be a reason for inability of the terminal nitrogen amino group to act as an efficient donor atom for hydrogen bonding. DFT- 
GIPAW (gauge including projected augmented wave), combined with the assignment of ${ }^{15} \mathrm{~N}$ shifts, corroborate the analysis of band population. The low-field signal at -27.2 and $-32.1 \mathrm{ppm}$ are attributed to $\mathrm{N} 2$ and $\mathrm{N} 1$ of $s$-tetrazine cycle, indicating a strong electron deficiency. Indeed, symmetric shielding components are strongly anisotropic with $\Delta \delta=140.0$ and $137.1 \mathrm{ppm}$, respectively. Consistently, spinning side bands (ssb; Figure $4 \mathrm{~b}$ and $4 \mathrm{c}$ ) are observed for only these signals, among the seven signals, although they exhibit the less intense signals. Noticeably, their principal components are oriented towards $\mathrm{N}=\mathrm{N}$ bond of triazole, supporting electronwithdrawing behavior. As $\mathrm{N} \mathrm{H}_{2}$ is involved into intramolecular hydrogen bonding, the corresponding ${ }^{15} \mathrm{~N}$ signal is shifted to lower field respecting to $\mathrm{N} 7$ amino group. The latter is, thus, able to participate in through-the-space charge transfer to $s$-tetrazine rings, in good agreement with our crystallographic analysis.

Vibrational properties. High dielectric constant organic semiconductors are of great importance for organic photovoltaic material research. ${ }^{39}$ Commonly, the light photon absorption generates electron-hole excitonic pair, in which electron and hole are bounded by Coulomb attraction. The potential of the latter is inversely proportional to the dielectric constant $\left(\varepsilon_{r}\right)$ of the material. Thus, the smaller potential is better for the charge separation, leading to the photocurrent increase. Otherwise, an electron-hole pair recombines into photon (radiatively or non-radiatively). The majority of organic semiconducting materials have $\varepsilon_{\mathrm{r}}=2-4$, that is far less than inorganic photovoltaic materials have. Therefore, the strong D-A bipolar nature of the IEF12 could offer unusual dielectric properties.

Under the framework of DFPT, it is possible to get the response of a solid on an imposed homogeneous electric field, which in due course, permits accessing the dielectric constant numerically. ${ }^{40,4}$ This procedure includes calculation of all $\Gamma$-phonons $(\boldsymbol{q}=0)$, which could be directly compared with our experimental vibrational spectroscopic data. For the present case, we used simple and rather accurate ATR-FTIR and confocal Raman spectroscopies to detect and compare the response of the material to a perturbation induced by probing electric field (Figure 
S4). The correlation plot of observed and calculated frequencies (Figure 5) obeys a linear dependency with a small intercept value, suggesting a good quality of the fit. All six components of dielectric tensor are independent for-1Laue class (Table 2).

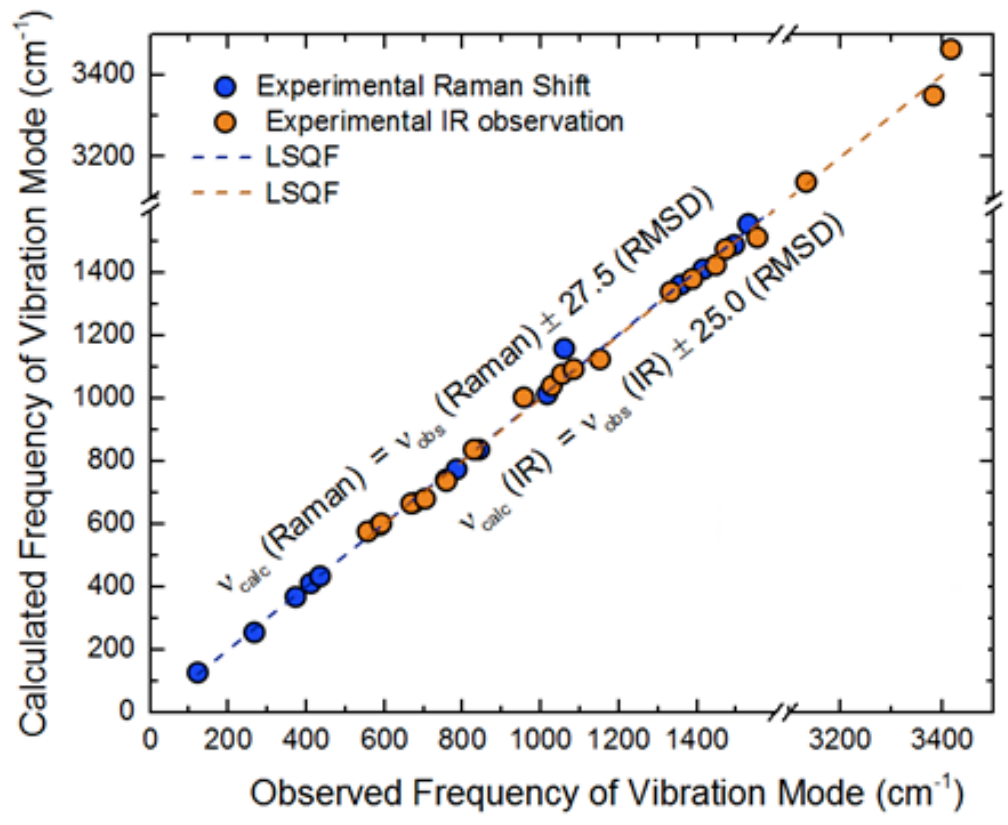

Figure 5. Correlation plot of observed (IR, Raman) and calculated vibration modes of IEF-12.

Table 2. Calculated independent components of (symmetry) static dielectric tensor and averaged value in polycrystalline state assumption.

\begin{tabular}{lccccccc}
\hline Dielectric tensor component & $\boldsymbol{\varepsilon}_{11}$ & $\boldsymbol{\varepsilon}_{12}$ & $\boldsymbol{\varepsilon}_{13}$ & $\boldsymbol{\varepsilon}_{22}$ & $\boldsymbol{\varepsilon}_{23}$ & $\boldsymbol{\varepsilon}_{33}$ & $\boldsymbol{\varepsilon}_{\text {poly }}$ \\
\hline Calculated value & 3.120 & -0.752 & 0.009 & 6.189 & -1.341 & 5.262 & 4.86 \\
\hline
\end{tabular}

The averaged value for polycrystalline IFE-12 is 4.86 , which is a very promising result for a small molecule organic semiconductor, ${ }^{39}$ even considering a maximal error of about $16 \%$, using the Perdew, Burke, and Ernzerhof (PBE) exchange-correlation functional benchmarked on inorganic semiconductors..$^{42}$ 
Solid state optical measurement and electronic band structure. Figure 6, depicting a usual Tauc plot, is addressed to determine optical bandgap of IEF-12. As it can be seen, the indirect transition takes place at $2.27 \mathrm{eV}$, corresponding to the deep-red shade of bulk sample (see picture inset). According to band structure analysis, conditional $T\left(0, \frac{1}{2}, 1 / 2\right) \quad \Gamma(0,0,0)$ pathway from valence band (VB) to conduction band (CB) defines a bandgap of $1.1 \mathrm{eV}$. Observed underestimation is known to be a limitation of the generalized gradient approximation (GGA), so we applied rigid shift of $\mathrm{CB}$ for comparison purposes. The visualization of orbitals (square of the absolute value of the wavefunction for a given electronic band, summed over all k-points) helps to recognize filled $\pi$-electron densities of DATR at the top of VB and empty $\pi$-states of $s$ tetrazine.

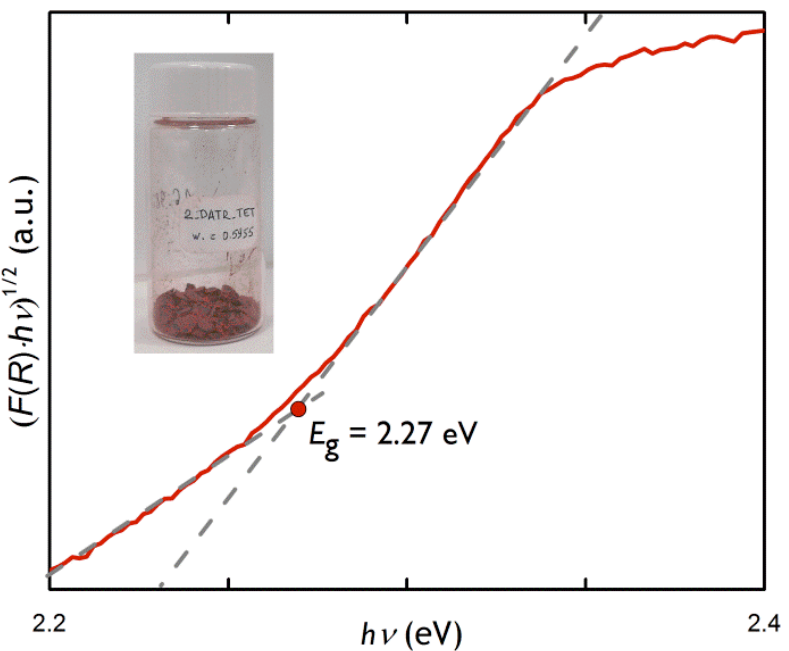

(a)

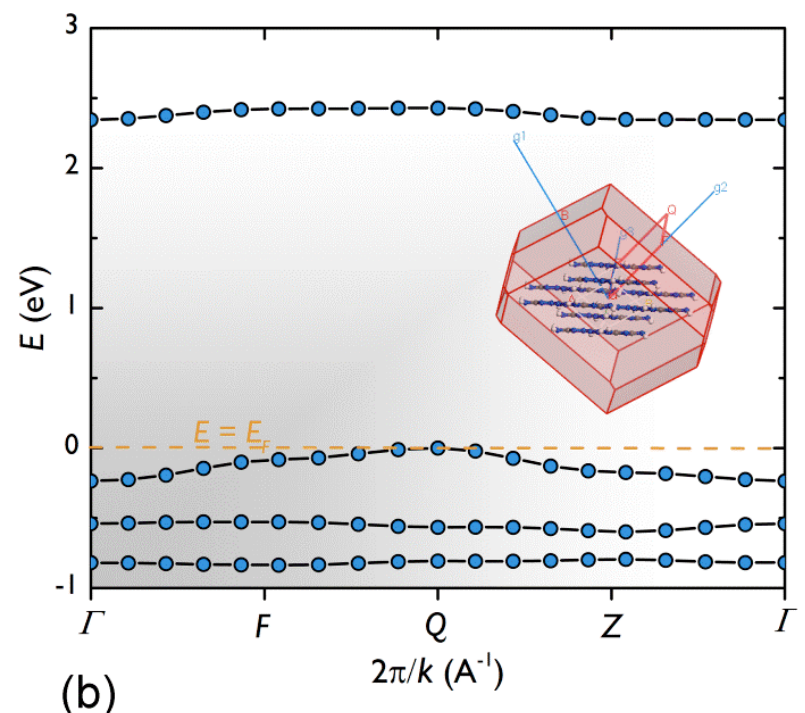

(b)

Figure 6. (a) Tauc plot of processed solid state optical absorption data (green and red dashed lines are given for the guide only), a view of prepared bulk sample is given in inset. (b) A frame of calculated band structure of IEF-12 superimposed with Wigner-Seitz cell (reciprocal vectors and Brillouin zone vectors are shown with blue and maroon lines).

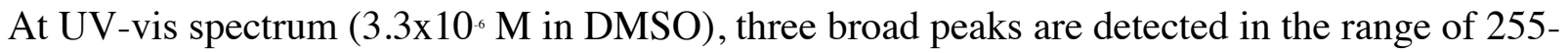
$600 \mathrm{~nm}$ (Figure S5). The low-energy band is centered at $520 \mathrm{~nm}\left(\varepsilon=84551 \mathrm{~mol}^{-1} \mathrm{~cm}^{-1}\right)$. The absorption bands at 332 and $285 \mathrm{~nm}$ are relatively intense by factor $\sim 25$ and $\sim 31$, respectively. 
The interpretation of optical transition can be greatly facilitated by TD-DFT, which is widely adopted for organic molecules of 20-200 atoms at reasonable accuracy and moderate calculation cost. ${ }^{43}$ Table 3 summarizes the main results of such calculations.

Table 3. Predicted vertical transition energies (singlets only), oscillator strengths in comparison to observed UV-vis absorption maxima.

\begin{tabular}{|c|c|c|c|c|c|}
\hline \multirow{2}{*}{ Excited state } & \multirow{2}{*}{ Transition } & \multicolumn{3}{|c|}{ TD-DFT } & \multirow{2}{*}{$\frac{\text { Experimental }}{\mathrm{nm}}$} \\
\hline & & $\mathbf{e V}$ & $\mathbf{n m}$ & $f$ & \\
\hline$\# 1$ & $\begin{array}{l}\text { HOMO-3 LUMO } \\
\text { HOMO LUMO }\end{array}$ & 2.14 & 579 & 0.0097 & 520 \\
\hline$\# \mathbf{3}$ & HOMO-2 LUMO & 2.46 & 545 & 0.0059 & \\
\hline$\# 4$ & $\begin{array}{l}\text { HOMO-3 LUMO } \\
\text { HOMO LUMO+1 }\end{array}$ & 3.60 & 344 & 0.0038 & \\
\hline$\# 6$ & $\begin{array}{l}\text { HOMO-3 LUMO } \\
\text { HOMO LUMO+1 }\end{array}$ & 3.80 & 327 & 0.7954 & 332 \\
\hline$\# 11$ & $\begin{array}{ll}\text { HOMO-16 } & \text { LUMO+1 } \\
\text { HOMO-7 } & \text { LUMO } \\
\text { HOMO-3 } & \text { LUMO+1 }\end{array}$ & 4.85 & 256 & 0.0254 & 284 \\
\hline$\# 13$ & $\begin{array}{l}\text { HOMO-1 LUMO+3 } \\
\text { HOMO LUMO+2 }\end{array}$ & 5.10 & 243 & 0.0012 & \\
\hline$\# 14$ & $\begin{array}{ll}\text { HOMO-7 } & \text { LUMO } \\
\text { HOMO-3 } & \text { LUMO+1 }\end{array}$ & 5.16 & 240 & 0.4287 & \\
\hline
\end{tabular}

The transition electron densities cannot be analyzed straightforwardly or by Kohn-Sham frontier orbitals (HOMO, LUMO at Figure 7), but their differences have more chemical sense. Thus, lower energy transitions $(\# 1, \# 6)$ are of $\pi^{*} \pi$ type, likely due to the depletion of electron density on 3,5-diamino-triazolyl rings and its accumulation on s-tetrazine cycle. The non- 
bonding states of nitrogen at triazole cycle donate electron density to the higher anti-bonding states of tetrazine also, resulting in an intense peak at $4.85 \mathrm{eV}$.
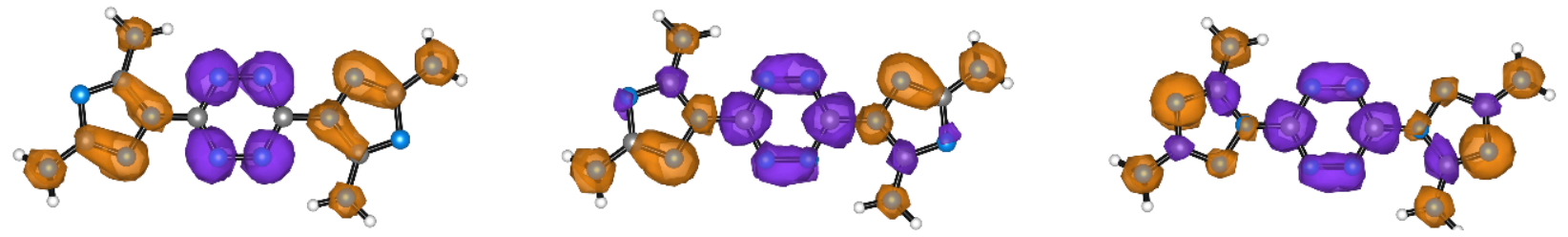

Figure 7. Electron densities difference of ground and excited state orbitals for most intense UVvis optical transitions.

Overall, these results agree with the solid state properties of IEF-12: i) the most interacting aggregate is the $\pi-\pi$ stacked system, and ii) population analysis of electronic bands reveals identical results, showing dominate occupation of VB with 3,5-diamino-triazole and CB with 1,2,4,5-tetrazine states.

\section{CONCLUSIONS}

This work points to the field of insensitive high energy as a potential source of small molecule organic semiconductors for optoelectronics (photoresistors, photodiodes, etc.). As exemplified on bis-3,5-diamino-1,2,4-triazolyl-1,2,4,5-tetrazine, such compounds can adopt the lamellar $\pi$ staking of trans-isomers, owing the combination of electron donor (3,5-diamino-triazole) and electron-withdrawing rings ( $s$-tetrazole), a relatively low bandgap and a higher dielectric constant, which is highly favorable for organic semiconducting materials.

For their practical use, small molecule or polymeric organic semiconductors are usually processed to thin films via wet methods (e.g. dip-, spin-coating, inkjet printing). However, in the present case, the strength of the H-bonds network (on par with other materials such as covalentorganic frameworks-COFs) strongly limits the solubility. Thus, based on our results, to facilitate the manufacturing and device fabrication of these organic semiconductors, chemical exfoliation ${ }^{4}$ or high vacuum evaporation route may be appropriate method for future thin film preparation. 


\section{ASSOCIATED CONTENT}

Supporting Information. The following files are available free of charge. SEM image of IEF-12, Cartesian coordinates of DFT optimized geometry of cis- and transisomers in a gas phase, details on Rietveld refinements of synchrotron PXRD and final crystallographic parameters summary, experimental ATR-FTIR and Raman spectra, PBC-DFT calculated vibrational frequencies, UV-vis absorption spectrum in DMSO and miscellaneous information.

\section{AUTHOR INFORMATION}

\section{Corresponding Author}

*patricia.horcajada@imdea.org

\section{Author Contributions}

The manuscript was written through contributions of all authors. All authors have given approval to the final version of the manuscript.

\section{Funding Sources}

The authors acknowledge RaPhuel (ENE2016-79608-C2-1-R). P. H. and A. A. B. are indebted to the People Programme (Marie Curie Actions) of the European Union's Seventh Framework Programme (FP7/2007-2013) under REA grant agreement no. 291803. P. H. also acknowledges the Ramón y Cajal RYC-2014-15039 (Ministerio de Ciencia, Investigación y Universidades).

\section{ACKNOWLEDGMENT}

The authors acknowledge RaPhuel (ENE2016-79608-C2-1-R). P. H. and A. A. B. are indebted to the People Programme (Marie Curie Actions) of the European Union's Seventh Framework 
Programme (FP7/2007-2013) under REA grant agreement no. 291803. P. H. also acknowledges the Ramón y Cajal RYC-2014-15039 (Ministerio de Ciencia, Investigación y Universidades). The authors would like to thank the European Synchrotron Radiation Facility (Grenoble, France)

and the French National Synchrotron Radiation Source "Synchrotron Soleil" (Saint-Aubin, France) for beamtime at the CRISTAL beamline.

\section{REFERENCES}

(1) Gao, H.; Shreeve, J. Azole-Based Energetic Salts. Chem. Rev. 2011, 111, 7377-7436 (see also references therein).

(2) Clavier, G.; Audebert, P. s-Tetrazines as Building Blocks for New Functional Molecules and Molecular Materials. Chem. Rev. 2010, 110, 3299-3314.

(3) Gong, Y. -H.; Miomandre, F.; Méallet-Renault, R.; Badré, S.; Galmiche, L.; Tang, J.; Audebert, P.; Clavier, G. Synthesis and Physical Chemistry of s-Tetrazines: Which Ones are Fluorescent and Why? Eur. J. Org. Chem. 2009, 2009, 6121-6128.

(4) Audebert, P.; Sadki, S.; Miomandre, F.; Clavier, G. First Example of an Electroactive Polymer Issued from an Oligothiophene Substituted Tetrazine. Electrochem. Commun. 2004, 6, $144-147$.

(5) Samanta, S.; Das, S.; Biswas, P. Photocatalysis by 3,6-Disubstituted-s-Tetrazine: VisibleLight Driven Metal-Free Green Synthesis of 2-Substituted Benzimidazole and Benzothiazole. $J$. Org. Chem. 2013, 78, 11184-11193.

(6) Samanta, S.; Ray, S.; Brata Ghosh, A.; Biswas, P. 3,6-Di(pyridin-2-yl)-1,2,4,5-Ttetrazine (pytz) Mediated Metal-free Mild Oxidation of Thiols to Disulfides in Aqueous Medium. RSC $A d v .$, 2016, 6, 39356-39363. 
(7) Chavez, D. E.; Hiskey, M. A.; Naud, D. L. Tetrazine Explosives. Propellants, Explos., Pyrotech. 2004, 29, 209-215.

(8) Kläpotke, T. M.; Preimesser, A.; Stierstorfer, J. Thermally Stable 3,6-Disubstituted 1,2,4,5Tetrazines. Z. Naturforsch. B, 2013, 68, 1310-1320.

(9) Chen, Y, Li, C.; Zhang, P.; Li, Y.; Yang, X.; Chen, L.; Tu, Y. Solution-Processable Tetrazine and Oligothiophene Based Linear A-D-A Small Molecules: Synthesis, Hierarchical Structure and Photovoltaic Properties. Org. Electron., 2013, 14, 1424-1434.

(10) Moral, M.; García, G.; Garzón, A.; Granadino-Roldán, J. M.; Fox, M. A.; Yufit, D. S.; Peñas, A.; Melguizo, M.; Fernández-Gómez, M. Electronic Structure and Charge Transport Properties of a Series of 3,6-(Diphenyl)-s-tetrazine Derivatives: Are They Suitable Candidates for Molecular Electronics? J. Phys. Chem. C, 2014, 118, 26427-26439.

(11) Moral, M; Garzón, A.; Olivier, Y.; Muccioli, L.; Sancho-García, J. C.; Granadino-Roldán, J. M.; Fernández-Gómez, M. Bis(arylene-ethynylene)-s-tetrazines: A Promising Family of nType Organic Semiconductors? J. Phys. Chem. C, 2015, 119, 18945-18955.

(12) Wang, C.; Dong, H.; Hu, W.; Liu, Y.; Zhu, D. Semiconducting $\pi$-Conjugated Systems in Field-Effect Transistors: A Material Odyssey of Organic Electronics. Chem. Rev. 2012, 112, 2208-2267.

(13) Madsen, I. C.; Hill, R. J. Collection and Analysis of Powder Diffraction Data with NearConstant Counting Statistics. J. Appl. Cryst. 1994, 27, 385-392.

(14) Shankland, K.; David, W. I. F.; Sivia; D.S. Routine Ab Initio Structure Determination of Chlorothiazideby X-ray Powder Diffraction Using Optimised Data Collection and Analysis Strategies. J. Mater. Chem. 1997, 7, 569-572. 
(15) Lee, C. T.; Yang, W. T.; Parr, R. G. Development of the Colle-Salvetti CorrelationEnergy Formula into a Functional of the Electron-Density. Phys. Rev. B, 1988, 37, 785-789.

(16) Krishnan, R.; Binkley, J.S.; Seeger, R; Pople, J.A. Self-Consistent Molecular Orbital Methods. XX. A Basis Set for Correlated Wave Functions. J. Chem. Phys. 1980, 72, 650-654.

(17) Woon, D. E.; Dunning Jr., T. H. Gaussian Basis Sets for Use in Correlated Molecular Calculations. III. The Atoms Aluminum through Argon. J. Chem. Phys. 1993, 98, 1358-1371.

(18) Weigend, F.; Ahlrichs, R. Balanced Basis Sets of Split Valence, Triple Zeta Valence and Quadruple Zeta Valence Quality for H to Rn: Design and assessment of accuracy. Phys. Chem. Chem. Phys., 2005, 7, 3297-305.

(19) Neese, F. The ORCA Program System. Wiley Interdiscip. Rev.: Comput.Mol. Sci. 2012, 2, 73-78.

(20) Hanwell, M. D.; Curtis, D. E.; Lonie, D.C.; Vandermeersch, T.; Zurek. E.; Hutchison, G. R. Avogadro: An Advanced Semantic Chemical Editor, Visualization, and Analysis Platform. $J$. Cheminformatics, 2012, 4, 17.

(21) Clark, S. J.; Segall, M. D.; Pickard, C. J.; Hasnip, P. J.; Probert, M. J.; Refson, K.; Payne, M. C. First Principles Methods Using CASTEP. Z. Kristallogr., 2005, 220, 567-570.

(22) Perdew, J. P.; Burke. K.; Ernzerhof, M.; Generalized Gradient Approximation Made Simple. Phys. Rev. Lett. 1996, 77, 3865.

(23) Tkatchenko, A.; Scheffler, M. Accurate Molecular van der Waals Interactions from Ground-State Electron Density and Free-Atom Reference Data, Phys. Rev. Lett. 2009, 102, 073005.

(24) Hamman, D. R. Optimized Norm-Conserving Vanderbilt Pseudopotentials. Phys. Rev. B: Condens. Matter Mater. Phys. 2013, 88, 085117. 
(25) Hamman, D. R. Erratum: Optimized Norm-Conserving Vanderbilt Pseudopotentials. Phys. Rev. B: Condens. Matter Mater. Phys. 2017, 95, 239906.

(26) Gonze, X. Perturbation Expansion of Variational Principles at Arbitrary Order. Phys. Rev. A: At., Mol., Opt. Phys., 1995, 52, 1086.

(27) Gonze, X. Adiabatic Density-Functional Perturbation Theory. Phys. Rev. A: At., Mol., Opt. Phys., 1995, 52, 1096.

(28) Pickard, C. J.; Mauri, F. All-electron Magnetic Response with Pseudopotentials: NMR Chemical Shifts, Phys. Rev. B, 2001, 63, 245101.

(29) Gervais, C.; Dupree, R.; Pike, K. J.; Bonhomme, C.; Profeta, M.; Pickard, C. J.; Mauri, F. Combined First-Principles Computational and Experimental Multinuclear Solid-State NMR, $J$. Phys. Chem. A, 2005, 109, 6960-6969.

(30) Chavez D. E.; Hiskey, M. A.; Gilardi, R.D.3,3 -Azobis(6-amino-1,2,4,5-tetrazine): A Novel High-Nitrogen Energetic Material. Angew. Chem. Int. Ed. 2000, 20, 1791-1793.

(31) Shlomovich, A.; Pechersky, T.; Cohen, A.; Yan, Q. L.; Kosa, M.; Petrutik, N.; Tal, N.;Aizikovich, A.; Gozin, M.Energetic Isomers of 1,2,4,5-Tetrazine-Bis-1,2,4-Triazoles With Low Toxicity. DaltonTrans. 2017, 46, 5994-6002.

(32) Parvari, G.; Levi, M.; Preshel Zlatsin, M.; Panz, L.; Grinstein, D.; Gottlieb, L; Denekamp, C.; Eichen, Y. Proposed Proton-Transfer Mechanism for the Initial Decomposition Steps of BTATz. J. Phys. Chem. A, 2018, 122, 5789-5798.

(33) Coelho, A. A. Indexing of Powder Diffraction Patterns by Iterative Use of Singular Value Decomposition. J. Appl. Cryst. 2003, 36, 86-95. 
(34) Altomare, A.; Corriero, N.; Cuocci, C.; Moliterni, A.; Rizzi, R. The Hybrid Big Bang-Big Crunch Method for Solving Crystal Structure from Powder Diffraction Data. J. Appl. Cryst. 2013, 46, 779-787.

(35) Rabie, U. M.; Salman, H. M. A.; Abou-El-Wafa, M. H. M. Charge-Transfer Molecular Complexes of Guanazole and Some 1,2,4-Triazoles with $\sigma$ - and $\pi$-Acceptors Involving a New Acceptor: Resulting In Situ; DDQH2. Bull. Chem. Soc. Jpn. 2004, 77, 681-686.

(36) Bernstein, J.; Davis, R. E.; Shimoni L.; Chang, N. -L. Patterns in Hydrogen Bonding: Functionality and Graph Set Analysis in Crystals. Angew. Chem. Int. Ed. 1995, 18, 1555-1573.

(37) Gavezzotti, A. Are Crystal Structures Predictable? Acc. Chem.Res. 1994, 27, 309-314.

(38) Ruiz, R; Choudhary, D.; Nickel, B.; Toccoli, T.; Chang, K. -C.; Mayer, A.C.; Clancy, P.; Blakely, J. M.; Headrick, R.L.; Iannotta, S.; Malliaras, G. G. Pentacene Thin Film Growth, Chem. Mater., 2004, 16, 4497-4508.

(39) Camaioni, N.; Po, R. Pushing the Envelope of the Intrinsic Limitation of Organic Solar Cells. J. Phys. Chem. Lett. 2013, 4, 1821-1828

(40) Gonze, X. First-Principles Responses of Solids to Atomic Displacements and Homogeneous Electric Fields: Implementation of a Conjugate-Gradient Algorithm. Phys. Rev. B, 1997, 55, 10337.

(41) Refson, K.; Tulip, P. R.; Clark, S. J. Variational Density-Functional Perturbation Theory for Dielectrics and Lattice Dynamics. Phys.Rev. B, 2006, 73, 155114.

(42) Petousis, I.; Chen, W.; Hautier, G.; Graf, T.; Schladt, T. D.; Persson, K. A.; Prinz, F. B. Benchmarking Density Functional Perturbation Theory To Enable High-Throughput Screening of Materials for Dielectric Constant and Refractive Index. Phys. Rev. B, 2016, 93, 115151. 
(43) Laurent, A. D.; Jacquemin, D. TD-DFT Benchmarks: A Review. Int. J. Quantum Chem.

2013, 113, 2019-2039

(44) Wang, H.; Zeng, Z.; Xu, P.; Li, L.; Zeng, G.; Xiao, R.; Tang, Z.; Huang, D.; Tang, L.; Lai, C.; Jiang, D.; Liu, Y.; Yi, H.; Qin, L.; Ye, S.; Ren, X.; Tang, W. Recent Progress in Covalent Organic Framework Thin Films: Fabrications, Applications and Perspectives. Chem. Soc. Rev., 2019, $48,488-516$.

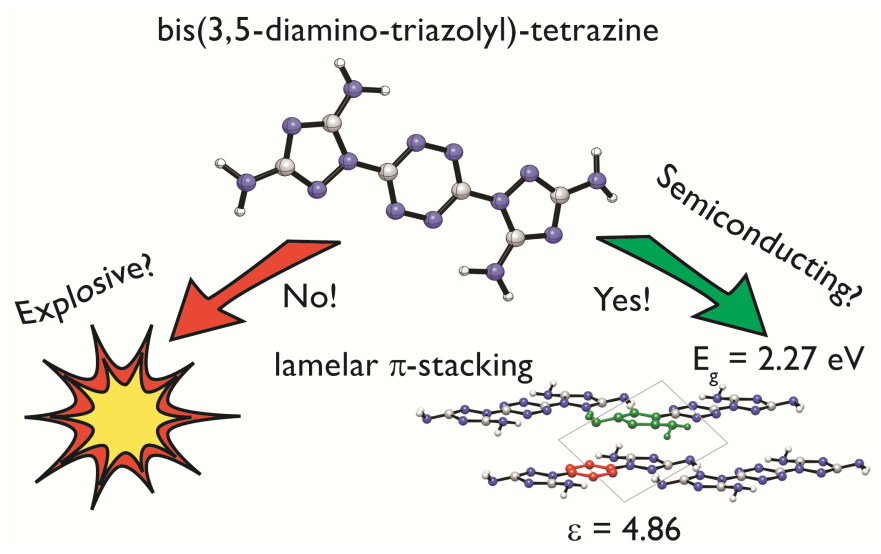

